Check for updates

Cite this: RSC Adv., 2019, 9, 6733

Received 24th December 2018 Accepted 18th February 2019

DOI: $10.1039 / c 8 r a 10540 e$

rsc.li/rsc-advances

\section{Novel triangular silver nanoparticle modified membranes for enhanced antifouling performance $\uparrow$}

\author{
Jabran Ahmad, Xianghua Wen, (ID * Fengjuan Li and Bo Wang
}

This study marks the first ever attempt at the successful fabrication of a novel reactive membrane to combat fouling through layer-by-layer (LBL) surface modification with polyelectrolyte (PE), followed by anisotropic triangular silver nanoparticles (TSNP). The morphology and the presence of TSNP on the membrane was confirmed by HR-TEM, FE-SEM and XPS. The charge density of the novel membrane (PE-TSNP) was increased 15.6 fold, as a result of the sharp-tip morphology of the TSNP forming tip-based "hot spots" on the membrane surface and high-atom-density active facets, which also enhanced the membrane hydrophilicity by $36 \%$. Owing to these improved features, the novel membrane displayed remarkable antibacterial and anti-adhesion properties by achieving 100\% bactericidal effect against high initial bacterial concentration $\left(10^{7} \mathrm{CFU} \mathrm{mL}{ }^{-1}\right)$. The membrane flux was improved by $31 \%$ while retaining a high flux recovery rate of $98.2 \%$ against biofouling. The membrane also mitigated organic and bio-organic fouling by maintaining high flux recovery rates of $96 \%$ and $95 \%$ respectively. As compared with a spherical silver nanoparticle modified membrane (PE-SSNP), the PE-TSNP membrane was $25.7 \%$ more hydrophilic and achieved $10 \%$ higher bacterial killing. Moreover, the novel membrane displayed $9.5 \%$, $11.6 \%$, and $14 \%$ higher flux recovery rates than that of the PE-SSNP membrane against biofouling, organic and bio-organic fouling respectively. Furthermore, the novel membrane retained a long-term biocidal capability of $93 \%$ even after 4 months of successive tests. ICP-MS revealed silver ion leaching of $4 \mu \mathrm{g} \mathrm{L}^{-1}$ and the total silver loss of $14 \%$ from the PE-TSNP membrane after 14 days.

\section{Introduction}

Polymeric membrane filtration has been widely considered as one of the most promising options for producing high quality and safe reclaimed water. However, membrane fouling is an unavoidable dilemma. The widespread nature of the microbes in wastewater and their tendency to adhere to the membrane surface can cause severe membrane biofouling. Furthermore, the natural organic matter (NOM) in wastewater streams causes severe membrane choking, leading to membrane waterflux decline. To combat fouling, some effective methods have been deployed by altering the surface charge or targeting the increased hydrophilicity of the membrane to discourage attachment of the foulants onto the surface., ${ }^{\mathbf{1 , 2}}$

Membrane surface modification with polyelectrolytes (PE) has proven efficient against carbon based organic foulants. Since many organic foulants are naturally hydrophobic, the PE modification impart a stronger hydrogen bond between the membrane and the surrounding water, constructing a protective

State Key Joint Laboratory of ESPC, School of Environment, Tsinghua University, Beijing 100084, China. E-mail: xhwen@tsinghua.edu.cn

$\dagger$ Electronic supplementary information (ESI) available. See DOI: 10.1039/c8ra10540e water layer from foulants..$^{3-5}$ Meanwhile, the clinging of the biofoulants can be mitigated by using antibacterial agents such as, metal nanoparticles (NP). ${ }^{6-8}$ In recent years, a large amount of research expertise has been devoted to membrane modification with various nanoparticles, where the modified membrane displayed high performance regarding anti-adhesion and antimicrobial properties. ${ }^{9-11}$ In several studies, membrane hydrophilicity and membrane water flux were increased due to the incorporation of nanoparticles onto the membranes. ${ }^{12,13}$

For the purpose of enhancing the antimicrobial activity, many metallic nanoparticles have been tested such as cesium, zinc, titanium, platinum, gold, and silver. Silver nanoparticles (AgNP) seem to outperform all other counterparts regarding the antimicrobial activity. It has been used as a disinfectant for centuries due to its efficient, nearly non-toxic effect on humans and tunable localized surface plasmon resonance (LSPR) properties. ${ }^{\mathbf{1 4 , 1 5}}$ To reduce organic and biological fouling, Diagne reported functionalized membrane surface with AgNP along with polyelectrolytes ${ }^{\mathbf{1 6}}$ while Mauter and coworkers conducted similar studies by utilizing AgNP and plasma treatment. ${ }^{17}$ Moreover, Agnihotri and coworkers have illustrated the size based antibacterial effect of spherical AgNP in solution. In their findings, the smallest AgNP size $(5 \mathrm{~nm}$ ) demonstrated the best results against all the tested strains ${ }^{18}$ 
Not only the size, but also the shape of metallic nanoparticles plays an important role in displaying remarkable and novel physicochemical properties. Advances in material science enabled the production of reactive anisotropic NP in a variety of different shapes like, spherical, rod, flag, nanotubes, flowershaped, cuboid, and triangle, etc. Each shape has its characteristics and different surface to volume ratio. Triangle silver nanoparticles (TSNP) are proven to be the most effective shape for antibacterial activity when tested along with other shapes of AgNP like rods, tubes and the spherical in suspension, thanks to the high-atom-density active facets $\{111\}$ of TSNP, which have direct interaction with bacterial cell. Furthermore, sharp vertex and edges of TSNP enable the NP to penetrate into the cell easily and damage the outer membrane. ${ }^{19}$ On the other hand, Hong and coworkers demonstrated Ag cubes with high antibacterial effect when compared with other shapes. ${ }^{20}$ Alshareef and group. Illustrated superior antibacterial effect of silver octahedral nanoparticles over spherical AgNP, and attributed geometric structure responsible for high performance. ${ }^{21}$

There is a clear lack of consistency between the published results on antibacterial effect of different shapes of AgNP, which was probably due to the lack of facile fabrication techniques to produce anisotropic particles with clear morphologies, and the difference between the fabrication routes adopted in different studies. However, all these results were in accord at the concept of anisotropic AgNP being superior antibacterial agent than that of isotropic AgNP (spherical AgNP). Furthermore, as many of the previous studies were conducted in solutions, they lack the possibility to form self-assembled structures and to study their prospect outcomes. Despite of the overwhelming evidence of anisotropic AgNP superiority, to date, all studies conducted for membrane modification used either spheres or roughly spherical NP either by adding NP in membrane fabrication process or used as surface coating agents.

Accordingly, we raised a hypothesis that a membrane with its surface modified using anisotropic triangular-shaped AgNP would be capable to display high antibiological and anti-organic fouling performance. Furthermore, to the best of our knowledge, there are no studies conducted yet which incorporate triangular-shaped AgNP for membrane modification to harness the potential of nanoparticles on the basis of their different shapes. This paper introduces the first ever attempt in delivering the novel pathway for the fabrication of reactive membrane via surface coating with TSNP onto the membrane surface. Membrane charged density was amplified via polyelectrolytes multilayer (PE) modification by using static layer-bylayer (LBL) method. Next, TSNPs were anchored firmly on the negatively charged polyelectrolyte matrix on the membrane surface. The method used is simple, versatile, reproducible and provide efficient control over membrane surface charge and coated nanoparticles concentration. TSNP modified membranes were compared with conventionally used spherical silver nanoparticles (SSNP) modified membranes. The modified membranes were tested for anti-adhesion, antimicrobial and fouling properties against $E$. coli and humic acid as model foulants. Shape-dependent modification imparted strong antibacterial and anti-adhesion activity to the membranes.
Moreover, novel membrane mitigated fouling by improving membrane water flux and demonstrated high flux recovery rate.

\section{Experimental section}

\subsection{Materials}

Silver nitrate $\left(\mathrm{AgNO}_{3}, \geq 99.98 \%\right)$, trisodium citrate $(\geq 99 \%)$, sodium hydroxide $(\mathrm{NaOH}, \geq 98 \%)$, sodium borohydride $\left(\mathrm{NaBH}_{4}, \geq 98 \%\right.$ ), humic acid (MW $=227.17$ ) were purchased from Aldrich. A $70 \mathrm{~W}$ sodium lamp was used to irradiate the mixture, was purchased from Osram China Lighting, China. Commercially available poly(ether sulfone) (PES) Microfiltration membrane was purchased from Corning, USA. Bacterial strain was obtained as stock culture from Sybrisk, China and cryopreserved in glycerol at $-18{ }^{\circ} \mathrm{C}$. Luria-Bertani (LB) nutrient agar from Aobox brand, China, was used for cultivation on solid medium. All culture media were sterilized by autoclaving at $120^{\circ} \mathrm{C}$ for $20 \mathrm{~min}$. All reagents were used as received and water from Millipore Milli-Q Purification system was used in the entire course of experiments.

\subsection{NP fabrication}

To illustrate superior anti-adhesion and anti-microbial activity of anisotropic TSNP, it was compared with widely used spherical silver nanoparticles (SSNP). To do so, SSNP of two different size range was fabricated, $4-6 \mathrm{~nm}$ and $\sim 30 \mathrm{~nm}$ were synthesized by using citrate reduction method. Briefly, for small size (4-6) SSNP of $\mathrm{AgNO}_{3}(250 \mu \mathrm{L}, 10 \mathrm{mM})$, trisodium citrate $(250 \mu \mathrm{L}, 100$ $\mathrm{mM}$ ) was poured in $25 \mathrm{~mL}$ of deionized water. Dropwise aqueous solution of $\mathrm{NaOH}(0.125 \mathrm{M})$ and $\mathrm{NaBH}_{4}(8 \mathrm{mM})$ was added into the solution. This solution was set under vigorous stirring at room temperature for $30 \mathrm{~min}$ in the dark, until the solution was homogenized and turned pale yellow. For larger size SSNP $(30 \mathrm{~nm})$ the concentration of $\mathrm{AgNO}_{3}$ was increased to $25 \mathrm{mM}$, while following the same procedure as adopted for small size SSNP. By simply varying the reactant concentration, while keeping the temperature and $\mathrm{pH}$ constant the size of the NP was controlled with great precision.

TSNP were fabricated by plasmon-mediated synthesis. The TSNP fabrication method was adopted from Xue et al. ${ }^{22}$ with the slight modification of the experimental setup, which resulted in fast production time at room temperature. Briefly, $\mathrm{AgNO}_{3}(250$ $\mu \mathrm{L}, 10 \mathrm{mM})$ and trisodium citrate $(250 \mu \mathrm{L}, 100 \mathrm{mM})$ was dispersed in $24.25 \mathrm{~mL}$ of deionized water under vigorous stirring at room temperature. Next, mixed aqueous solution of $\mathrm{NaOH}(0.125 \mathrm{M})$ and $\mathrm{NaBH}_{4}(8 \mathrm{mM})$ was poured dropwise into the former solution. The resultant silver seeds were immediately subjected to irradiation with a $70 \mathrm{~W}$ sodium lamp, mounted into indigenously made Styrofoam triangular hood with its interior wall lined with aluminum. The aluminum lining served as a heat sink for the little heat generated by the lamp. This set up facilitated more kinetically controlled reaction by controlling the heat generated during irradiation. The reaction mixture was irradiated for $90 \mathrm{~min}$. Air inlet was provided to ensure the regular supply of oxygen necessary for NP shape transformation. 


\subsection{NP characterization}

As prepared NP were characterized by using UV-Vis spectrometry UV-3101PC scanning spectrophotometer (Shimadzu, Japan) with a $1 \mathrm{~cm}$ quartz cell. The morphology and particle size of nanoparticle was analyzed by high-resolution transmission electron microscopy (HR-TEM, Tecnai G20, FEI, USA) under an accelerating voltage of $200 \mathrm{kV}$. The samples were prepared by placing a drop on the surface of 400-mesh carbon-coated $\mathrm{Cu}$ grid and dried under sodium lamp for ten min before examining. X-ray diffraction (XRD) was performed to verify the NP crystalline structure and presence of facet planes (Panalytical Xpert-PRO 3050/60). Samples were prepared by concentrating TSNP solution through centrifugation at $15000 \mathrm{rpm}$ for $12 \mathrm{~min}$. The supernatant was removed from the sample. The process was repeated three times. Next, concentrated solution was added dropwise on the glass slide and allowed to dry at room temperature.

\subsection{Membrane surface modification}

The commercially available poly(ether sulfone) (PES) microfiltration membrane (MF) coupon $(0.1 \mu \mathrm{m}$ pore size $)$ was placed erected in a holder with the top membrane surface exposed for treatment. Two polyelectrolytes (PE) namely, poly styrene sulphone (PSS) solution as anion $\left(0.02 \mathrm{~mol} \mathrm{~L}^{-1}, \mathrm{MW}=70000\right.$, Aldrich Chemical Co. USA) and positively charged poly (diallyldimethylammonium chloride) (PDADMAC) solution (0.02 $\mathrm{mol} \mathrm{L}^{-1}, \mathrm{MW}=200$ 000, 350 000, Aldrich Chemical Co. USA) were used and membranes were subjected to layer-by-layer (LBL) modification. PE was alternately deposited on the top of membrane surface for three min, with DI water rinse in between the layers. Layering anion followed by cation (PSS + PDADMAC) makes 1 bilayer. Each layer act as a surface primer for the next oppositely charged layer. In this work, after achieving 1 bilayer, an additional terminal anion (PSS) was deposited. The resulting 1.5 bilayer was achieved with each layer comprises of approximately $5 \mathrm{~nm}$ in thickness with the top most layer negatively charged by PSS to maximize the negative surface charge of the membrane. The thickness of the PE layers was determine using EP3 single-wavelength multi-angle imaging ellipsometer, (nanofilm). After desired PE layers coatings, the membranes were modified with two different shapes of NP separately i.e. with TSNP (average size $30 \mathrm{~nm}$ ) and with SSNP (4-6 nm and $\sim 30$ $\mathrm{nm}$ ) to examine the shape-dependent anti-adhesion and antimicrobial performance of the as prepared membranes. Although, it is already established that the smaller size AgNP has better antimicrobial efficacy, but for the sake of uniformity in the particle size between SSNP and TSNP, the membranes were also modified by using SSNP size of $\sim 30 \mathrm{~nm}$ with $\mathrm{Ag}$ content of $2 \mathrm{wt} \%$, and were tested in parallel. The characterization and filtration performance of $30 \mathrm{~nm}$ PE-SSNP2 membrane is summarized in ESI (Fig. S6b, $\dagger$ Table 1). Membranes were obtained with three different silver weight percentage (wt\%) $0.5 \mathrm{wt} \%, 1 \mathrm{wt} \%$ and $2 \mathrm{wt} \%$ respectively. The resulting membranes were named (PE-TSNP) polyelectrolytestriangular silver nanoparticles modified membrane and (PESSNP) polyelectrolytes-spherical silver nanoparticles modified membrane, with numeric suffix of $0.5,1$ and 2 indicating the wt\% of Ag (PE-TSNP 0.5, PE-TSNP1, PE-TSNP2, PE-SSNP0.5, PE-SSNP1, PE-SSNP2).

\subsection{Membrane characterization}

To verify the presence of $\mathrm{Ag}$ on the membrane surface, the membranes were analyzed by field emission scanning electron microscope (FE-SEM; Sigma, Zeiss) equipped with energy dispersive X-ray (EDX) spectrometer. $\mathrm{Ag}$ wt\% and elemental state of $\mathrm{Ag}$ was examined by X-ray photoelectron spectroscopy (XPS) (ULVAC-PHI 1800, Japan) with an Al K $\alpha$ (1486.6 eV) radiation source and a high-resolution spectrum were collected for the Ag 3d peaks. The take-off angle of the photoelectron was set to $75^{\circ}$. Membrane hydrophilicity was determined by contact angle measurements via sessile drop method with video supported interface (OCA 20, Dataphysics, Germany). Modified membranes were immediately tested for change in contact angle. Each measurement was performed in triplicate to ensure high accuracy. Eight readings were conducted for each membrane, and the results were taken as average. The membrane surface charge was analyzed using electrokinetic analyzer SurPass 3 (Anton Paar, Austria) with $1 \mathrm{mM} \mathrm{KCl}$ solution at $\mathrm{pH}$ value of $5.3 \pm 0.3$. The zeta $(\zeta)$ potential of as-prepared nanoparticles and size distribution was measured by using Malvern instruments. Membrane surface roughness and mean pore size was determined by atomic force microscopy (AFM). To examine a possible change in pore size upon modification, the pore size of the membranes before and after PE modification was determined by taking high-resolution images of the small area in conjunction with digital store line profile. The line provides a mean to quantify the holes on the membrane surface. The results are the average of 40 pores measurement for each membrane.

Table 1 Contact angle, $\zeta$ potential and initial flux data of the virgin and modified membranes

\begin{tabular}{|c|c|c|c|c|}
\hline & Virgin membrane & PE-modified & PE-SSNP2 modified & PE-TSNP2 modified \\
\hline Contact angle (degree) & $35.6 \pm 2.66$ & $33.3 \pm 3.61$ & $32 \pm 1.72$ & $24.7 \pm 2.48$ \\
\hline$\zeta$ potential $(\mathrm{mV})$ & $-3.56 \pm 0.52$ & $-48.6 \pm 0.92$ & $-49.8 \pm 0.42$ & $-55.6 \pm 0.84$ \\
\hline Mean pore size $(\mathrm{nm})$ & $99 \pm 0.127$ & $91 \pm 0.186$ & - & - \\
\hline Initial flux (biofouling) $\left(\mathrm{L} \mathrm{m}^{-2} \mathrm{~h}^{-1}\right)$ & $93 \pm 2.2$ & $99 \pm 3.1$ & $104.6 \pm 2.4$ & $127 \pm 2.6$ \\
\hline Initial flux (organic fouling) $\left(\mathrm{L} \mathrm{m}^{-2} \mathrm{~h}^{-1}\right)$ & $90 \pm 1.2$ & $91 \pm 0.83$ & $98 \pm 1.6$ & $110 \pm 2.2$ \\
\hline Initial flux (bio-organic fouling) $\left(\mathrm{L} \mathrm{m}^{-2} \mathrm{~h}^{-1}\right)$ & $85 \pm 0.63$ & $90 \pm 0.94$ & $93 \pm 1.8$ & $108 \pm 1.9$ \\
\hline
\end{tabular}




\subsection{Anti-adhesion activity of PE-TSNP and PE-SSNP modified membranes}

E. coli was used as model microorganism to evaluate the antiadhesion activity of the modified membranes. The bacterium inoculum was prepared by cultivating bacterial strain in LB nutrient agar and incubated at $37^{\circ} \mathrm{C}$ for $24 \mathrm{~h}$. Initial bacterial concentration was set to $10^{7}$ colony forming unit per milliliter $\left(\mathrm{CFU} \mathrm{mL}{ }^{-1}\right)$. Isolated colonies were mixed in $1 \%(\mathrm{w} / \mathrm{v})$ aqueous saline solution. Both modified membranes (PE-TSNP modified and PE-SSNP modified) were first sterilized in autoclave at $121{ }^{\circ} \mathrm{C}$ for $5 \mathrm{~min}$. The sterilized membranes were submerged in a Petri dish containing $40 \mathrm{~mL}$ of LB medium. Aliquots of $300 \mu \mathrm{L}$ of bacterial inoculum was added to each dish containing PETSNP and PE-SSNP separately. Membranes were exposed to

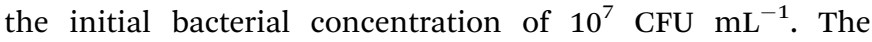
samples were incubated at $37{ }^{\circ} \mathrm{C}$ for $36 \mathrm{~h}$ without stirring. Immediately after incubation period, membranes were removed and rinsed with $1 \%(\mathrm{w} / \mathrm{v})$ aqueous saline solution to remove non-adhered cell. The membranes were then transferred into $20 \mathrm{~mL}$ sterile $1 \%(\mathrm{w} / \mathrm{v})$ aqueous saline solution and was set to sonication for $20 \mathrm{~min}$. $100 \mu \mathrm{L}$ aliquots were taken from the resulting suspension and was serially diluted before plated on LB. The LB plates were incubated at $37{ }^{\circ} \mathrm{C}$ for $24 \mathrm{~h}$. Subsequently, the number of grown colonies were counted and adhered cells was expressed as CFU $\mathrm{mL}^{-1}$. The final result was plotted in terms of loss viability percentage by using equation:

$$
\text { Loss viability }(\%)=\frac{n}{N} \times 100
$$

where $n$ is the number of bacterial cells (CFU) in the modified membranes and $N$ is the CFU value of the control (virgin PES membrane).

\subsection{Antibacterial activity}

To illustrate the shape dependent antimicrobial activity of the anisotropic NP modified membranes, cross comparison between PE-TSNP and PE-SSNP was carried out by using standard plate count method. Bacterial inoculum was prepared as describes in Section 2.6, and initial bacterial concentration was set to $10^{7} \mathrm{CFU} \mathrm{mL}{ }^{-1}$. Aliquots of $500 \mu \mathrm{L}$ of bacterial inoculum was added to a flask containing $50 \mathrm{~mL}$ LB nutrient agar. Modified sterile membranes were then subjected to the suspension and incubated at $37{ }^{\circ} \mathrm{C}$ for $24 \mathrm{~h}$. After incubation period, $100 \mu \mathrm{L}$ of the suspension was taken and serially diluted in peptone water $1 \%(\mathrm{w} / \mathrm{v})$ and plated on LB nutrient medium for $24 \mathrm{~h}$. The virgin and PE modified membranes were used as control. TEM analysis was performed to examine the treated bacteria for possible cell damage.

\subsection{Ag leaching}

Modified membranes were tested for Ag leaching periodically to access the stability of the coated NP layer. Membranes were soaked in $20 \mathrm{~mL}$ of DI water for 14 days. Samples were collected on daily basis and DI water was changed every $24 \mathrm{~h}$. Inductively coupled plasma mass spectrometry (ICP-MS) was used for the $\mathrm{Ag}$ ions quantification. All samples were digested by using concentrated nitric acid at pH 2 before subjected to ICP-MS analysis.

\subsection{Filtration studies}

Filtration experiments were conducted to evaluate the modified membrane performance against fouling. A dead-end stirred cell with a total cell volume of $50 \mathrm{~mL}$ (Amicon model 8050, Millipore Corp.) with an effective membrane area of $13.4 \mathrm{~cm}^{2}$, was connected to a high pressure nitrogen tank, through a stainless steel pressure reservoir of $10 \mathrm{~L}$. The pure water flux $(J)$ of the membranes was measured at an applied pressure of $60 \mathrm{kPa}$. The permeate was collected at determined intervals and the flux was calculated by using the equation:

$$
J=\frac{W}{A t}
$$

where $W$ is the weight of the permeate collected (kg), $A$ is the membrane effective area $\left(\mathrm{cm}^{2}\right)$ and $t$ is the permeation time (h).

All filtration experiment consisted of three successive steps. For biological fouling; at first, the pure water flux $\left(J_{0}\right)$ was measured by filtering DI water via membrane. Second, the $E$. coli suspension with a concentration of $10^{7} \mathrm{CFU} \mathrm{\textrm {mL } ^ { - 1 }}$ in phosphate buffer saline (PBS) solution was filtered for $150 \mathrm{~min}$ and the flux was recorded $\left(J_{\mathrm{b}}\right)$, followed by membrane unloading

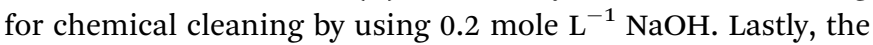
chemically cleaned membrane was remounted into the filtration cell and the pure water flux was measured again, which is denoted by $\left(J_{\mathrm{f}}\right)$. For organic fouling, humic acid solution $\left(20 \mathrm{mg} \mathrm{L}^{-1}\right.$ ), and for the combined fouling the mixture of humic

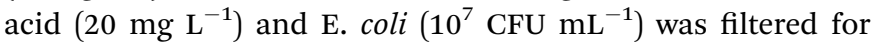
$150 \mathrm{~min}$. The pure water flux was of the fouled membrane was measured after the membranes were chemically cleaned with 0.2 mole $\mathrm{L}^{-1} \mathrm{NaOH}$ and were reloaded into the filtration cell to measure the pure water flux $\left(J_{\mathrm{f}}\right)$.

In this study the term "flux recovery' $(J \%)$ is referred to the difference between initial pure water flux before fouling $\left(J_{0}\right)$ and pure water flux after fouling and cleaning $\left(J_{\mathrm{f}}\right)$ as calculated by eqn (3) and used to evaluate the membrane performance. All the filtration tests were performed in triplicates and the results were expressed as the average plus errors:

$$
J \%=\frac{J_{0}-J_{\mathrm{f}}}{J_{0}} \times 100
$$

\section{Results and discussion}

\subsection{Characterization of the as prepared TSNP}

UV-visible spectroscopy is considered to be the most widely used technique for nanoparticles characterization. The absorption spectrum (Fig. 1a) of pale yellow nanoparticles solution prepared by citrate reduction method showed a surface plasmon resonance (SPR) band with a maximum of $\sim 395 \mathrm{~nm}$, indicating the presence of spherical AgNP. SPR dictates the optical absorption spectra of metal NP which shift to longer wavelength with increased particle size. Multiple peaks were observed for TSNP prepared by plasmon-mediated synthesis as 
(a)

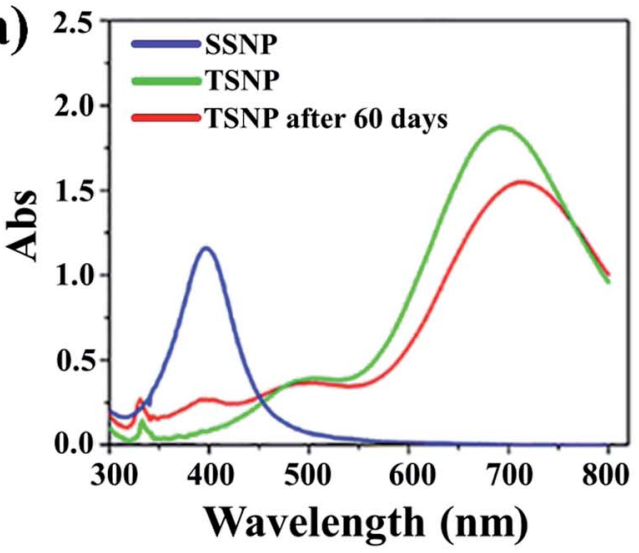

(b)

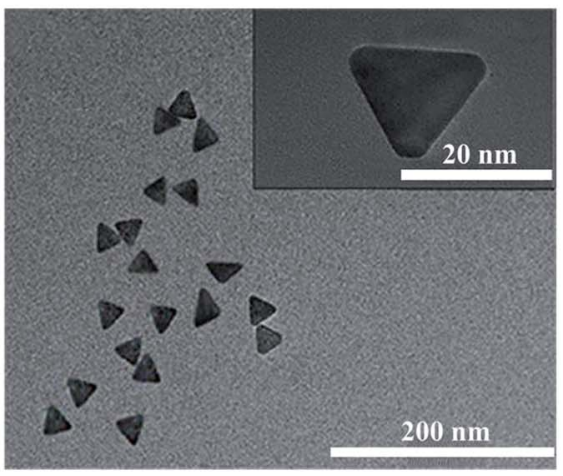

(c)

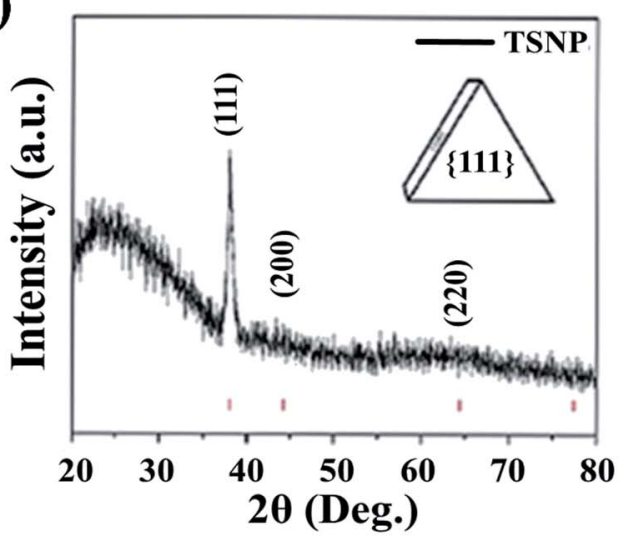

(d)

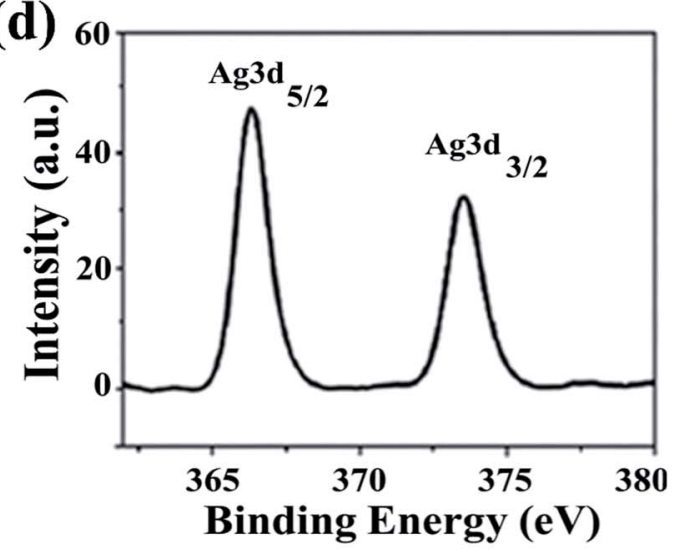

Fig. 1 (a) Absorption spectrum of spherical silver nanoparticles and triangular silver nanoparticles. (b) HR-TEM image of the triangular silver nanoparticles. The inset shows the morphology of the single triangular silver nanoparticle. (c) XRD spectra of TSNP. Planes at $\{111\},\{200\},\{220\}$ indicates face-centered cubic ( $f(c)$ lattice structure of crystalline Ag. (d) Shows the XPS spectrum of the PE-TSNP2 modified membrane. Distinct $\mathrm{Ag} 3 \mathrm{~d}$ peaks indicating the presence of $\mathrm{Ag}$ on the membrane surface.

anisotropic NP give rise to more than one absorption peaks due to their asymmetrical orientation. The peaks at $\sim 520 \mathrm{~nm}$ and $\sim 690 \mathrm{~nm}$ shows the characteristics of typical TSNP, which correspond to the out-of-plane quadrupole, the in-plane quadrupole, and the in-plane dipole plasmon resonance modes of the TSNP. Fabrication of sharp-tip TSNP is of great importance as the electric field is high at the tips of TSNP which enhance the activity of the particle. The TSNP obtained displayed sharp tip morphology. Chen and Carroll ${ }^{23}$ calculated resonance peak for a perfect TSNP at $\sim 770 \mathrm{~nm}$ which resides very close to the as prepared TSNP. Plasmon-mediated synthesis provided well defined and uniform triangles. Peaks obtained are in accordance with the work of Xue et al. ${ }^{22}$ Long reaction time lead to the larger particle size and also particles aggregation was noticed (data not shown).

To illustrate the surface morphology and size of nanoparticles, high resolution transmission electron microscope was used, which revealed an average TSNP size of $\sim 30 \mathrm{~nm}$ (Fig. 1b) and SSNP size range from 4 to $6 \mathrm{~nm}$ (Fig. 3c) respectively. Samples were loaded on the carbon-coated copper grid and dried under sodium lamp. TSNP obtained through plasmon mediated synthesis showed clear triangular morphology. Moreover, TSNPs were highly monodispersed, and no aggregation of particles was observed.
3.1.1. Crystal structure of the TSNP. XRD analysis of the TSNP was performed by Bruker D8 advanced X-ray diffractometer, using $\mathrm{CuK} \alpha$ radiation $(\lambda=1.5418 \AA)$, under $40 \mathrm{kV} / 30 \mathrm{MA}-\mathrm{X}$ ray, $2 \theta / \theta$ scanning mode. Data were obtained for the $2 \theta$ range of 20 to 80 degrees. Theoretically, the high intense peak for cubic material is (111) reflection, which was clearly observed in the prepared TSNP. As shown in the Fig. 1c, four peaks at $2 \theta$ values of $38.116,44.227,64.426$, and 77.472 degree. corresponding to (111), (200), (220) and (311) planes of Ag were observed and compared with the standard powder diffraction card of JCPDS, silver file no. 04-0783. The X-ray diffraction measurement illustrated the remarkably intensive diffraction of the characteristic peak at a $2 \theta$ value of 38.11 corresponds to the reflections of metallic TSNP crystallized in the face-centered cubic (fcc) structure with basal $\{111\}$ lattice planes. A slower reduction rate due to the heat control during the reaction and low $\mathrm{pH}$ value, resulted in a slow reaction rate. The resultant low concentration of silver atoms favoured the silver atoms deposition on the (100) facets and led to the formation of $\{111\}$ faceted TSNP.

3.1.2. NP stability. Nanoparticles stability is of keen importance as Ag-based NP are highly susceptible to oxidation, thus losing their integrity. To ensure the NP stability, the as prepared TSNP were subjected to two different conditions (stored at $4{ }^{\circ} \mathrm{C}$ and room temperature) for 60 days UV-Vis 
spectrometry results (Fig. 1a) confirmed that the particles retained their morphology and monodispersity after stored at different conditions for 60 days. TSNP stability can be ascribed to the high $\zeta$ potential value of $-24.08 \mathrm{mV}$ (SSNP $\zeta$ potential $=$ $-21.84 \mathrm{mV}$ ). Anisotropic NP possess numerous edges, large surface area and resultant high negative charge as compared to isotropic NP. ${ }^{24}$

\subsection{Membrane modification}

3.2.1. TSNP on membrane surface as illustrated by XPS and FE-SEM. Fig. 2 shows the PES membrane modification steps. The membranes were first coated by PE (PSS, PDADMAC) to achieve the desired 1.5 bilayer followed by either coating with TSNP $(\sim 30 \mathrm{~nm})$ or SSNP (4 to $6 \mathrm{~nm})$. AFM results showed that the mean pore size (MPS) of the PE-modified membrane was barely effected upon modification (Table 1) due to the inherent large pore size of PES microfiltration membrane and the lower number of PE layers used in this study. XPS, is a surface sensitive technique which was employed to analyze the presence of immobilized NP onto the membrane surface. Data shown in Fig. 1d, illustrates the high resolution Ag 3d XPS spectrum of the PE-TSNP2 modified membrane with the binding energies of $\mathrm{Ag} 3 \mathrm{~d} 3 / 2$ and $\mathrm{Ag} 3 \mathrm{~d} 5 / 2$ at $373.8 \mathrm{eV}$ and $367.7 \mathrm{eV}$, respectively. The results suggest that the Ag present on membrane surface is metallic Ag. No corresponding peaks were found for lattice oxygen $\mathrm{O}_{2}$ from $\mathrm{Ag}_{2} \mathrm{O}$. As $\mathrm{Ag}$ can easily be oxidized by oxygen, it is possible that part of Ag from PE-SSNP modified membranes was oxidized, as particle size used was very small. However, data obtained from XRD and XPS indicates that the predominant part of $\mathrm{Ag}$ species was $\mathrm{Ag}(0)$.

FE-SEM revealed the decoration of TSNP on membrane surface. The membrane modified with $0.5 \mathrm{wt} \% \mathrm{Ag}$, showed lower membrane surface coverage. But, higher Ag wt $\%$ (3.5 wt\%) showed NP aggregation on membrane (Fig. S1a and
$\mathrm{S} 1 \mathrm{~b} \dagger)$. The results can be interpreted as $\mathrm{Ag}$ content of $0.5 \mathrm{wt} \%$ to be low to cover the membrane surface appropriately. Hence, in the later part of the studies only PE-TSNP1 and PE-TSNP2 are addressed. Fig. 3a illustrate the PE-TSNP2 membranes displayed highly mono dispersed decoration of TSNPs over the entire membrane surface. Few particles were found in the membrane pores. Moreover, many TSNPs were arranged in tipto-tip manner in PE-TSNP2 which leads to the formation of socalled "hot spots" (Fig. 3b). In metal NP self-assemblies, different plasmon modes can interact with each other by near-field coupling, when the interparticle distance is smaller and when there is a uniformity among the two or more plasmonic nanostructures, owing to the transfer and confinement of electromagnetic energy. Thus only uniform TSNP can be closely packed in forming hot spots. These hot spots are known to drastically enhance the electric field between the tips. ${ }^{25}$ The hot spots and the resultant high electric charge have shown their effect in increased membrane hydrophilicity and membrane flux enhancement which are further discussed in the later sections.

3.2.2. Membrane hydrophilicity and zeta potential. Membrane hydrophilicity was evaluated by contact angle measurement, which confirmed the increased in hydrophilicity upon modification with $\mathrm{PE}$ and $\mathrm{Ag}$ nanoparticles (Table 1). Despite the fact that PES microfiltration membranes have inherently high permeability and hydrophilicity, the PE-TSNP2 modified membranes were $36 \%$ more hydrophilic than virgin membranes. Increased membrane hydrophilicity of PE-TSNP2 membranes can be attributed to the highly charged tips of TSNPs which increased the charged density of the membrane. Membrane hydrophilicity can be enhanced by increasing the number of PE layers on the membrane surface, the trait which can be harnessed when addressing salts and ions rejection or to develop selective barriers and rejection capabilities. ${ }^{26}$ In this study, the number of bilayers were restricted to 1.5 bilayer to

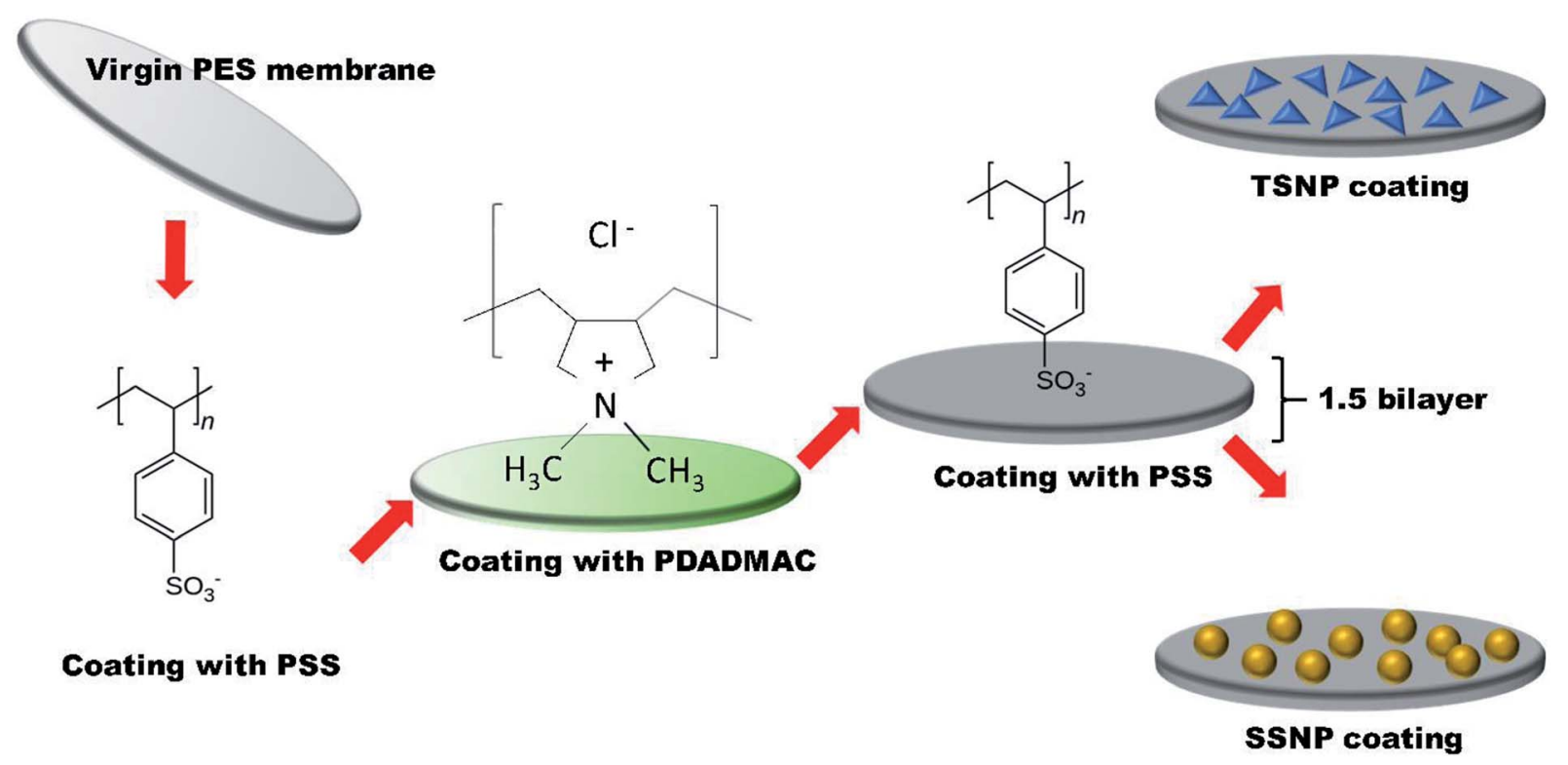

Fig. 2 Schematic illustration of membrane modification steps by using polyelectrolytes and triangular/spherical Ag nanoparticles. 
(a)

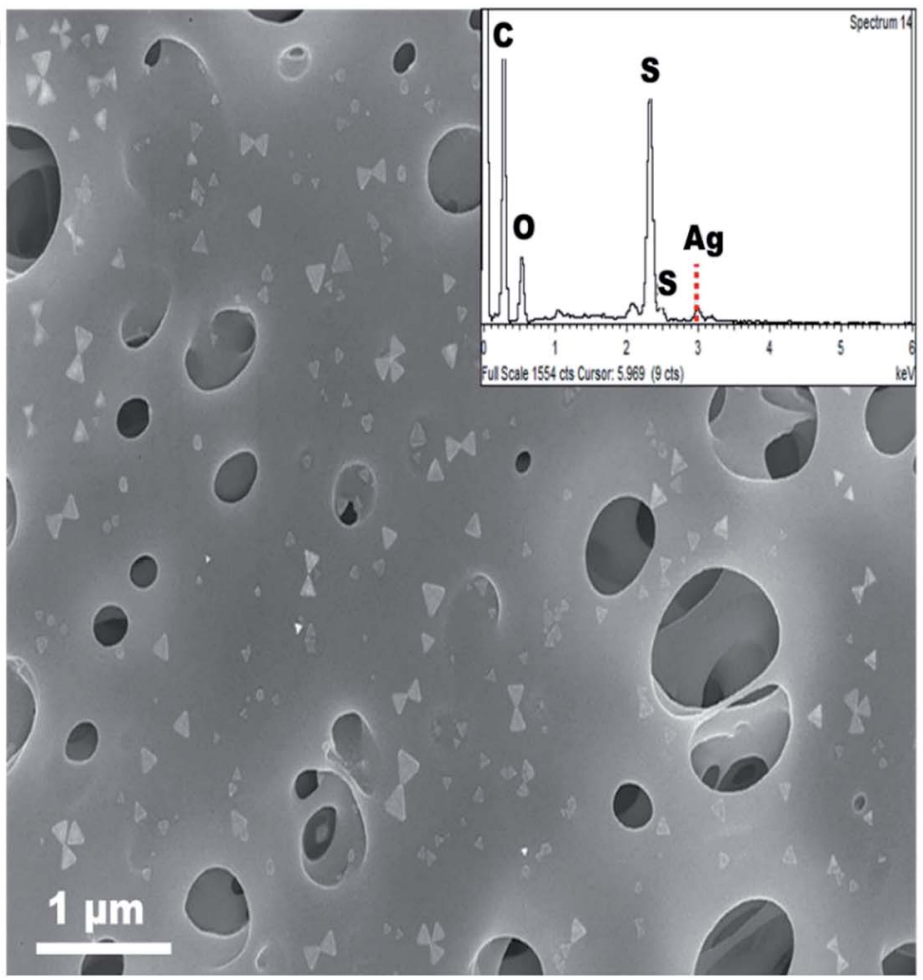

(b) Hot spots formation

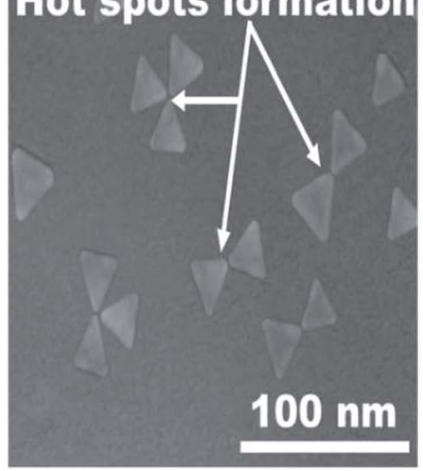

(c)

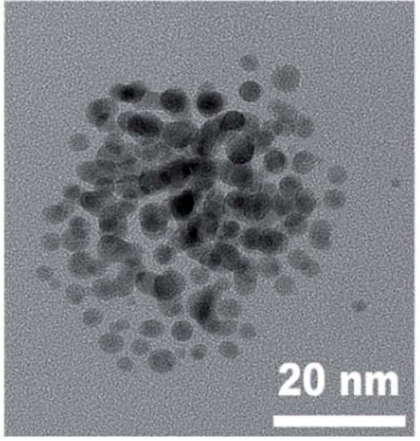

(d)

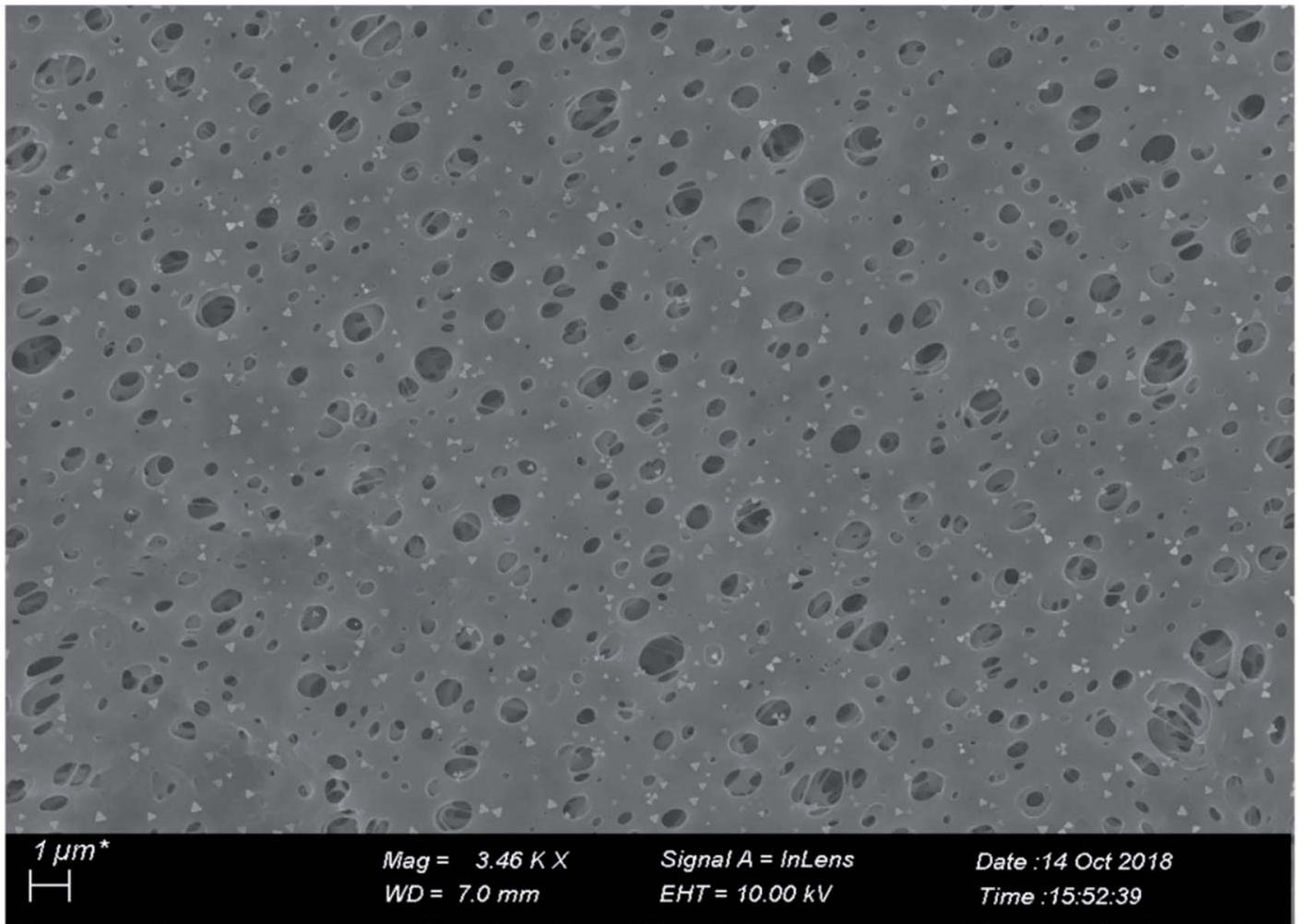

Fig. 3 (a) FE-SEM image of PE-TSNP2 modified membranes at high magnification (Mag =17.36 K $\times$ ), (inset) EDS spectra of PE-TSNP2 membrane (b) TSNP forming hot spots on the membrane surface (c) HR-TEM image of spherical AgNP (d) FE-SEM image of PE-TSNP2 membrane, covering large surface of the membrane at lower magnification.

avoid compromise on membrane pore size and the membrane flux, which increased membrane surface charged density to 12.6 fold upon modification with $\mathrm{PE}$, as compare to virgin PES membrane (Table 1) which is appropriate to anchor NP firmly onto the membrane surface without compromising membrane permeability. Furthermore, slight increase in membrane $\zeta$ 
potential was observed upon SSNP and TSNP incorporation. Higher $\zeta$ potential value of TSNP might translated into higher membrane surface $\zeta$ potential of PE-TSNP modified membranes, which further increased the membrane net charge to 15.6 folds. The resultant highly charged PE-TSNP2 membrane was $25.7 \%$ more hydrophilic than that of PESSNP2 membrane. Ellipsometeric data showed each PE layer thickness of $\sim 5 \mathrm{~nm}$ at two different $\mathrm{pH}$ values ( $\mathrm{pH} 7, \mathrm{pH}$ 9) and the resulting decrease in contact angle upon increasing the number of PE layers (Fig. S2a and S2b, Note $1 \dagger$ ).

3.2.3. Ag ions leaching from the membrane. In this study, the $\mathrm{Ag}$ ion release rate was examined for a PE-TSNP2 membrane for 14 days. Ag leaching from the membrane showed an initial abrupt decline and then displayed steady decline rate after 6 days (Fig. 4). Total silver ion release after 14 days on average was accounted only for $4 \mu \mathrm{g} \mathrm{L}^{-1}$, which is well below the silver maximum contaminants limit $(100 \mu \mathrm{g}$ $\mathrm{L}^{-1}$ ) mandated by World Health Organization (WHO) and U.S. Environmental Protection Agency (USEPA). PE-SSNP2 membrane showed silver leaching of $5 \mu \mathrm{g} \mathrm{L}^{-1}$. The possible reason behind the slight Ag leaching increase for PE-SSNP2 membrane is that the smaller Ag particles are more susceptible to oxidation in comparison with large Ag particles due to their lower redox potential. ${ }^{27}$ Due to the higher reduction potential of $\mathrm{O}_{2}\left(E_{0}=1.23 \mathrm{~V}\right)$, small silver NP oxidize by $\mathrm{O}_{2}$ faster with their lower redox potential $\left(E_{0}=0.8 \mathrm{~V}\right) .{ }^{28} \mathrm{Based}$ on the initial silver concentration on the PE-TSNP2 modified membrane the cumulative silver leaching from the membrane was $14 \%$ after 14 days which illustrated that the modified membranes retained large quantities of silver after leaching experiment; thus, can provide relatively long-term biocidal effect during the life span of the membrane. Yin and coworkers documented similar leaching pattern. In their studies the amount of silver leached out during 14 days was $12 \%$ of the initial silver concentration on the membrane $^{29}$

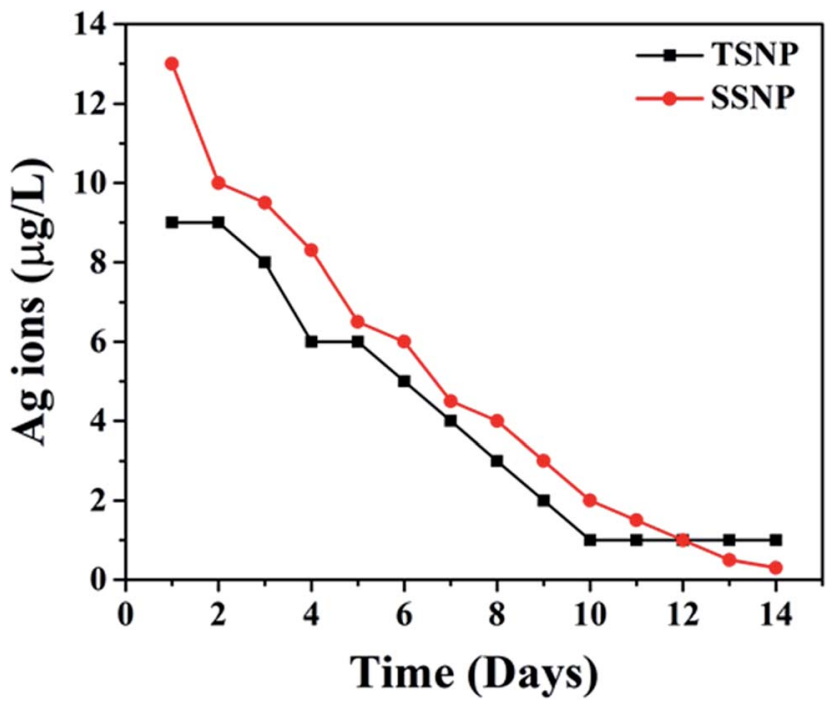

Fig. $4 \mathrm{Ag}$ ions leaching from PE-TSNP2 and PE-SSNP2 modified membrane.

\subsection{Antibacterial performance}

The antimicrobial activity of PE-SSNP and PE-TSNP modified membranes was evaluated against $E$. coli. The PE-TSNP2 modified membranes showed remarkable antibacterial capabilities by displaying almost $100 \%$ bactericidal activity. All the samples treated with PE-TSNP2 showed zero cell growth as can be seen in Fig. 5. As expected, PE-SSNP1 and PE-SSNP2 membranes achieved only $82 \%$ and $91 \%$ disinfection with the exposed bacterial concentration thus displayed inferior performance as compared to PE-TSNP modified membranes. PETSNP2 membranes displayed 10\% higher bacterial killing than that of PE-SSNP2 membranes. It is noteworthy, that PETSNP1 also achieved higher bactericidal activity (97\%) than PE-SSNP2, which showed that in comparison with SSNPs, very little amount of TSNPs are ample to display high bactericidal effect. Moshe and group reported only $75 \%$ bacterial reduction by using spherical silver nanoparticles of $15 \mathrm{~nm}$ diameter on RO membrane, ${ }^{30}$ while Wen and coworkers demonstrated membrane modification using spray and spin assisted layer-bylayer deposition of copper nanoparticles for antifouling and antibacterial purpose. With 10 bilayers the modified membranes achieved $94 \%$ bacterial mitigation. ${ }^{31}$ Nanoparticle size plays a crucial role in antimicrobial effect. Ag particles of size $5 \mathrm{~nm}$ or less are considered to possess very lethal antimicrobial capabilities by providing large surface area for attachment. ${ }^{32} \mathrm{AgNP}$ of size $4 \mathrm{~nm}$ formed the major portion of SSNP used in this study (Fig. 3c and S3†), which is the ideal candidate in the family of spherical AgNP to be used for antibacterial purpose. However, TSNP outperformed SSNP by exhibiting enhanced disinfection rate against high bacterial concentration. In other studies, the TSNP obtained were largely truncated. ${ }^{33,34}$ In this work, TSNP were successfully fabricated with sharp tips morphology. The sharp tips of TSNP have the capability to damage the bacterial cell by disrupting membrane integrity, as can been seen in Fig. 5b. In the studies conducted by Pal et $a l .{ }^{33}$ similar disinfection pattern was found where TSNP in solution displayed $100 \%$ disinfection against $E$. coli when compared with other shapes of silver nanoparticles in suspension (spherical and rods).

3.3.1. Anti-adhesion performance. The microbial adhesion to the membrane surface is a prerequisite for biofilm formation. Bacteria after clinging to the membrane surface proliferate and form a cake layer which leads to membrane fouling and hampers membrane performance. Membrane surface modification has an advantage over modifying the entire thickness of the membrane as most of the interaction with microbes occur on the membrane surface where the NP surface is also exposed to perform the antimicrobial activity. Hence, producing membrane with reactive surface is of keen importance. To illustrate the anti-adhesion capabilities of the membranes, PETSNP2 and PE-SSNP2 were exposed to LB medium containing $E$. coli for $36 \mathrm{~h}$. The adhered cells were plate counted and expressed as percentage inhibition using virgin membrane as control. Fig. 5a demonstrates the superior anti-adhesion performance of PE-TSNP2 modified membrane. No visual growth was observed on the PE-TSNP2 membranes. In contrast, 
(a)

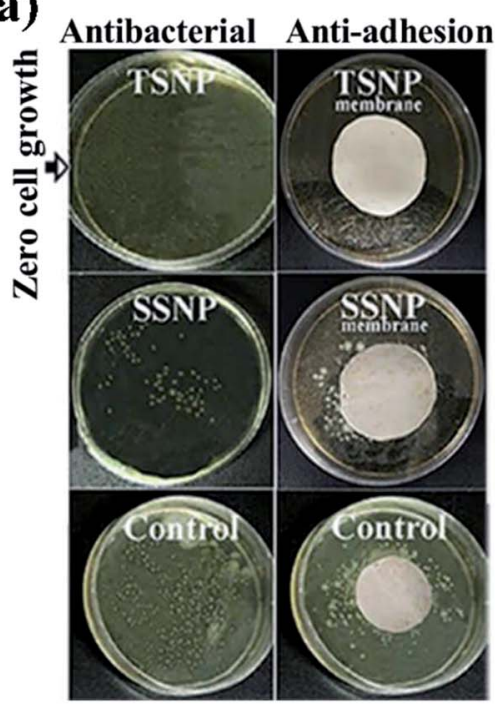

(b)

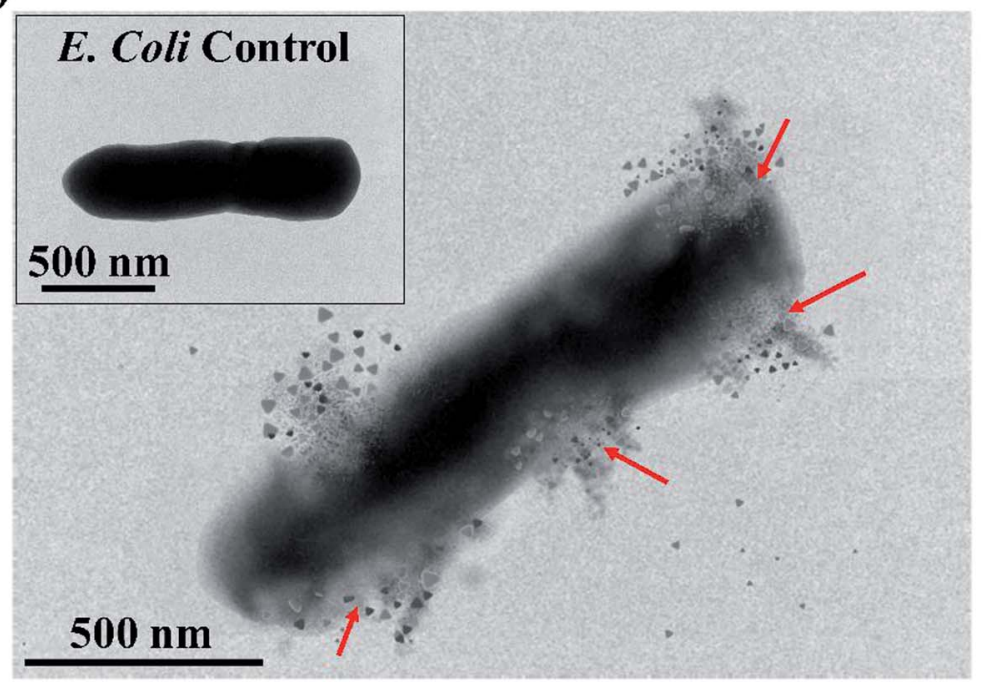

Fig. 5 Bacterial killing results, (a) plate count results of PE-SSNP2 and PE-TSNP2 modified membranes initially supplemented with $10^{7} \mathrm{CFU}_{\mathrm{mL}}{ }^{-1}$ of $E$. coli for antibacterial and anti-adhesion studies. Membranes photograph provided the visual evidence of the bacterial growth contrast of two membranes used. Virgin PES membrane was used as control (b) TEM image of $E$. coli physical mutilation upon TSNP contact. Arrows indicating membrane damage. Inset image shows healthy E. coli cell as control.

PE-SSNP2 membranes were able to reduce the number of adhered cells to $79.3 \pm 3.4 \%$. The differences observed in the antibacterial and anti-adhesion activities between membranes modified with two different shapes of AgNP can be attributed to the NP morphology percent active facets. Along with the sharp tip morphology, the top basal plane of TSNP is a $\{111\}$ surface, which is a high-atom-density facet. While SSNP are dominated by $\{100\}$ facets. In our work, enhanced activity of the TSNP loaded membrane can be attributed to the presence of highatom-density active $\{111\}$ facets. $\{111\}$ facets are found to have direct interaction with bacterial cell as describes by Morones et $a l .{ }^{35}$ Pal et $a l .^{33}$ also attributed $\{111\}$ active facets to be responsible for enhance TSNP antibacterial activity. Furthermore, an increase in membrane surface $\zeta$ potential by PE layers and further increase in charge upon TSNP coating might translated into strong electrostatic repulsion between membrane surface and $E$. coli cells.

3.3.2. Membrane filtration performance. For $\mathrm{MF}$ membrane, the most common type of fouling is biofouling, which is a multi-step process where bacteria, biopolymers and proteins attach or absorb on a membrane surface and can block membrane pores. Biofouling is a major hindrance in membrane usage, because unlike other types of fouling, the microorganisms can grow, multiply, relocate and secrete extracellular polymeric substances (EPS). This multi-step adsorption of substances and the proliferation of microbes forms a fully developed biofilm on the membrane surface. ${ }^{36}$ Fig. 6 a indicate the initial and recovered flux after 150 min of filtration of all the membranes used against biofouling. All the modified membranes displayed flux enhancement as compared to unmodified PES membrane. PE-SSNP2 membrane showed $11.83 \%$ increase in initial flux, while PE-TSNP1 showed 9\% increase. PE-TSNP2 displayed the highest initial flux with 36\% increase in comparison to neat PES membrane and 21\% higher than PE-SSNP2 membranes. The increase in permeation flux of the modified membranes can be ascribed to the increase in membrane surface hydrophilicity by PE and AgNP coating, which is supported by contact angle and membrane surface $\zeta$ potential measurement results (Table 1). Higher flux of PETSNP2 can be attributed to the high charge density on the PETSNP2 membrane, imparted by highly charged tips of TSNP and the resultant high electric field generation due to the formation of "hot spots". Raman spectra (Fig. S4†) confirmed the high electric field enhancement of self-assembled plasmonic TSNP forming hotspots. The performance of the unmodified PES membrane was severely hampered by biofouling. Fig. $6 \mathrm{~b}$ indicate the flux recovery rate of neat PES membrane which was $83.8 \%$. Very little improvement was observed upon modification with PE only $(84.7 \%)$ while PESSNP2 achieved flux recovery rate of $89.6 \%$. On the other hand, the flux recovered by PE-TSNP1 and PE-TSNP2 was 96.1\% and $98.2 \%$ respectively. The results were in accordance with antibacterial activity test where similar trend was noticed (Fig. 5a). As bacterial fouling is the surface phenomenon and usually results in reversible cake layer, if the attachment of the bacteria to the membrane surface is not strongly bounded. The increased negative charge of the membrane upon PE modification discourage the bacterial attachment on the membrane surface while TSNP cause killing-on-contact with their highatom-density facets hence, mitigating the bacterial proliferation at large on the membrane surface. The reversible deposition of bacteria can be easily be removed from the modified membrane surface, thereby resulting in lower flux decline. It is noteworthy, that PE-TSNP1 membrane held 50\% less Ag weight content than that of PE-SSNP2 membranes, but still exhibited superior performance.

3.3.3. Organic fouling. Organic foulants cause fouling by depositing on internal and external membrane surface. Usually 
(a)

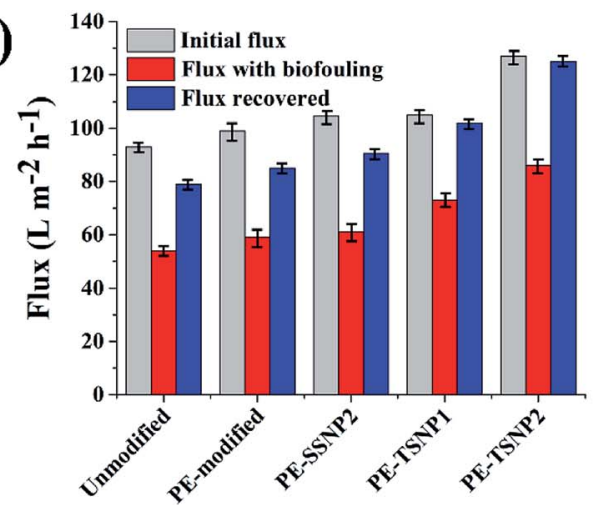

(c)

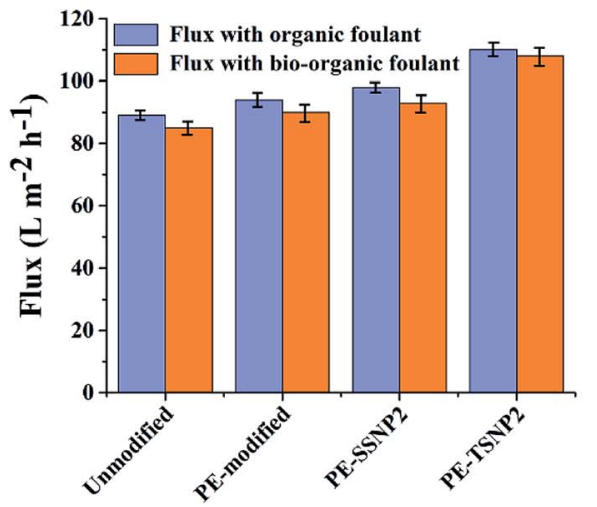

(b)

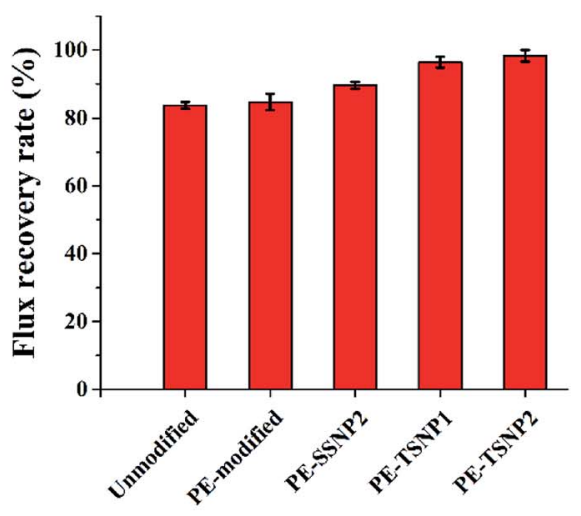

(d)

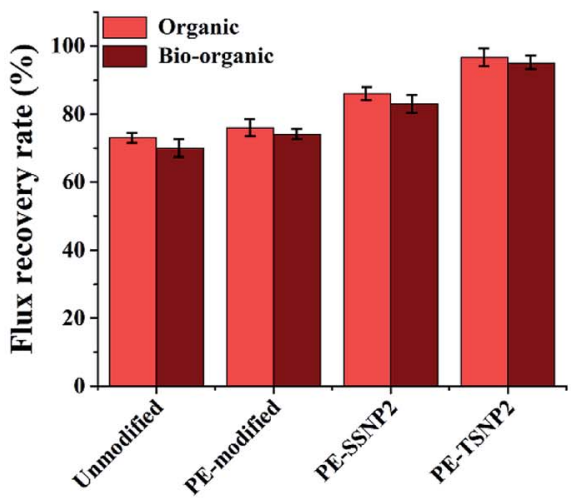

Fig. 6 (a) Initial flux, flux during biofouling and flux after fouling and cleaning of all the membranes used. (b) Flux recovery rate percentages of the membranes with biofouling. (c) Initial flux during organic and bio-organic fouling (d) flux recovery rate with organic and bio-organic fouling.

adsorption is considered as a main fouling mechanism behind organic fouling, which cause surface pore blocking or cake layer formation. The reversible cake layer is formed when foulants with large molecular weight are prevented from entering into the pores due to their large size. However, small solute particles can be easily penetrated into the membrane pore and cause irreversible fouling. ${ }^{37,38}$

Organic fouling experiments were conducted at relatively high organic concentration $\left(20 \mathrm{mg} \mathrm{L}^{-1}\right)$. The membranes modified with $2 \mathrm{wt} \% \mathrm{Ag}$ for both PE-TSNP and PE-SSNP were selected to examine the organic and bio-organic fouling, because of their better performance against biofouling. All the modified membranes displayed increase in initial flux (Fig. 6c). The flux enhancement was observed upon PE modification. The PE-TSNP2 membranes showed the highest initial flux, i.e. 22\% and $15.7 \%$ higher flux than that of virgin and PE-SSNP2 membranes. Similarly, Fig. 6d shows the flux recovery rates of the membranes. PE-TSNP2 membranes achieved the highest flux recovery rate of $96 \%$. While PE-SSNP2 membrane recovered 86\%. PE-TSNP2 membranes demonstrated $11.6 \%$ higher flux recovery rate than PE-SSNP2 membranes. Virgin membrane suffered greatly with organic fouling with its flux recovery rate of only $73 \%$. The enhancement in flux recovery rate followed the trend with increased in hydrophilicity of the membranes. As the membrane become more hydrophilic, the fouling was retarded. The higher the hydrophilicity of the membrane, the higher was the flux recovery rate. This may be due to the electrostatic repulsion imparted by hydrophilic charged surface of the membrane, which hinders the humic acid attachment on the membrane surface.

3.3.4. Bio-organic fouling. Against bio-organic fouling, all the membranes showed almost similar initial flux (Fig. 6c), like it was noticed for organic fouling, which indicated that the fouling using both components (humic acid and $E$. coli) is dominated by organic fouling. Almost similar results of flux recovery rate was achieved for bio-organic fouling (Fig. 6d), as they were displayed in organic fouling experiments. PE-TSNP2 membrane showed the highest flux recovery rate of $95 \%$, which was $23 \%$ and $14 \%$ higher than that of virgin and PESSNP2 membranes. Studies performed by Diagne illustrated $8.9 \%$ flux decline against bio-organic fouling, while in this work, only $5 \%$ of the initial flux was lost. ${ }^{16}$ The research conducted by Jian and group showed that membranes modified with silver NP achieved $90 \%$ flux recovery rate against organic fouling. ${ }^{39}$ Virgin membrane was only able to recover $70 \%$ of its initial flux. The reason behind the slightly lower initial flux and the flux recovery rate during organic fouling may be due to the poly dispersion and variation in size range of humic acid ( 0.4 to $3.3 \mathrm{~nm}$ ) which can travel into the membrane pore and cause irreversible fouling. ${ }^{40}$ Thus, the portion of organic fouling was larger than that of biological fouling. Enhanced performance of PE-TSNP2 membranes can be attributed to the increased electric field by the plasmonic self-assembled TSNP. Raman spectra showed the high electric field enhancement by self-assembled TSNP (Fig. S4 $\dagger$ ) decorated on the membrane surface, which improved the contact angle and zeta potential of the membrane, 
(a)

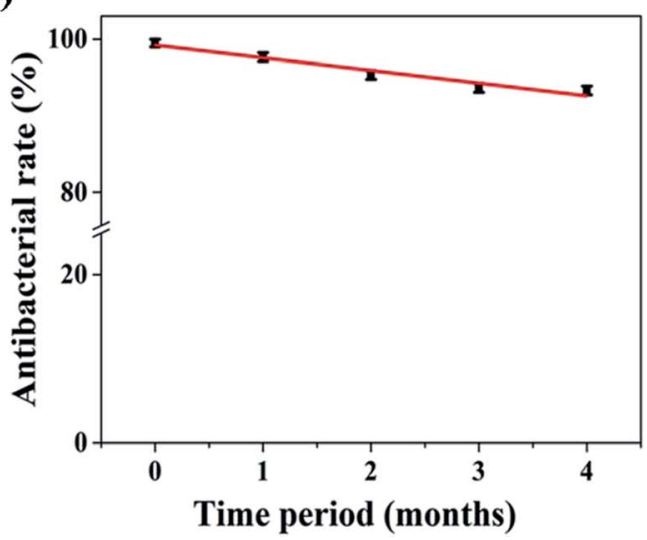

(b)

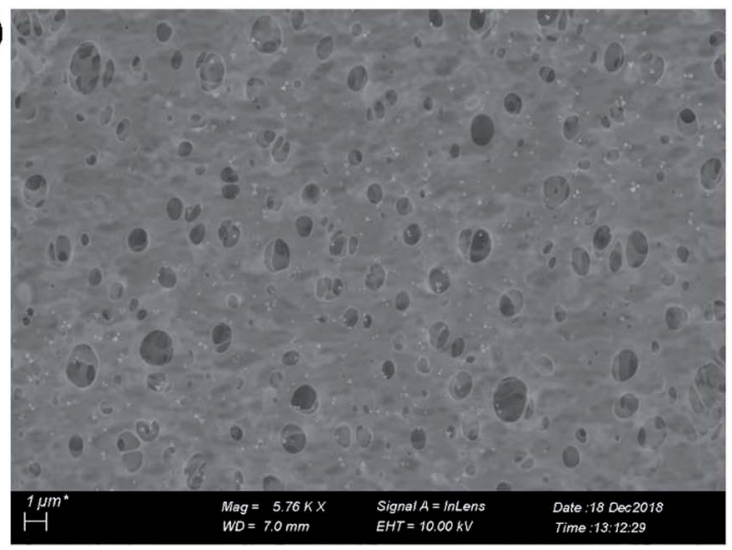

Fig. 7 Long-term membrane performance (a) antibacterial rate of the PE-TSNP2 modified membranes against E. coli. (b) FE-SEM image of PETSNP2 membrane after fouling in long-term studies, showing the presence of triangular silver nanoparticles on the membrane.

consequently, reduced the hydrophobic interaction between the surface of the membrane and humic acid. Moreover, the surface roughness of the PE-TSNP2 membrane was found to be lower in comparison with PE-SSNP2 (Fig. S5†), which also assisted in fouling mitigation.

3.3.5. Long-term biocidal studies. The gradual leaching of $\mathrm{Ag}$ from the membrane poses the threat of eventual loss of biocidal capabilities of the modified membrane in the long-run. As a result, the long-term stability of the PE-TSNP2 modified membrane was tested for four months. After each encounter with $E$. coli, the tested membranes were rinsed thoroughly with Milli-Q water, dried and stored at room temperature. Fig. 7 shows that the membrane retained $93 \%$ of antibacterial effect even after four month of successive test, ensuring the long-term stability of the PE-TSNP2 modified membranes.

\section{Conclusion}

Commercially available PES membranes were successfully modified by polyelectrolytes (PDADMAC, PSS) and triangular silver nanoparticles. The modified membrane displayed remarkable shape-dependent antibacterial, anti-adhesion and anti-fouling a performance against $E$. coli. In comparison with spherical silver nanoparticles modified membranes, which only achieved $91 \%$ bacterial killing and were able to reduce the number of adhered cells to $79.3 \pm 3.4 \%$, the PE-TSNP modified membranes demonstrated $100 \%$ antibacterial killing efficiency with high bacterial concentration $\left(10^{7} \mathrm{CFU} \mathrm{mL}^{-1}\right)$. Novel PETSNP membranes showed improved membrane hydrophilicity and flux enhancement by $36 \%$ and $31 \%$ and achieved high flux recovery rate of $98.2 \%$ against biofouling. PE-TSNP membrane also proved robust against organic and bio-organic fouling by maintaining the high flux recovery rate of $96 \%$ (organic fouling) and $95 \%$ (bio-organic fouling). Silver leaching remained limited to only $4 \mu \mathrm{g} \mathrm{L}^{-1}$ after 14 consecutive days of the experiment. Long-term membrane performance was also evaluated. The PETSNP membranes retained $93 \%$ of antibacterial effect even after four months of successive test. The PE-TSNP membranes displayed shape dependent antibacterial activity due to the superior traits of triangular silver nanoparticles, which possess high-atom-density active facets. Along with polyectrolytes, the sharp-tip anatomy of TSNP forming "tip-based hot spots" on the membrane surface, resulted in increased negative membrane surface charge which imparted electrostatic repulsive force between bacteria and membrane surface, resulting in enhanced anti-adhesion performance and reduced biofouling.

The study conducted here can provide a benchmark in producing novel reactive membranes with enhanced antibacterial and anti-biofouling capabilities. This work will also help in sensitizing the nanoparticles production industry on focusing more in the production of anisotropic nanoparticles in bulk, which will lower down their cost and will eventually be a preferred choice for membrane modification and antibacterial applications.

\section{Conflicts of interest}

There are no conflicts to declare.

\section{Acknowledgements}

This research was supported by the International Cooperation Program of MOST of China (2016YFE0118800) and the Major Water Project of China (2014ZX07216).

\section{References}

1 R. Van Houdt and C. W. Michiels, J. Appl. Microbiol., 2010, 109, 1117-1131.

2 S. Wang, T. Li, C. Chen, B. Liu and J. C. Crittenden, Front. Environ. Sci. Eng., 2018, 12, 3.

3 G. dong Kang and Y. ming Cao, Water Res., 2012, 46, 584600.

4 V. Kochkodan and N. Hilal, Desalination, 2015, 356, 187-207.

5 J. Xu, Z. Wang, J. Wang and S. Wang, Desalination, 2015, 365, 398-406.

6 L. Gzara, Z. Ahmad Rehan, S. B. Khan, K. A. Alamry, M. H. Albeirutty, M. S. El-Shahawi, M. I. Rashid, A. Figoli, 
E. Drioli and A. M. Asiri, J. Taiwan Inst. Chem. Eng., 2016, 65, 405-419.

7 L. Tang, K. A. Huynh, M. L. Fleming, M. Larronde-Larretche and K. L. Chen, J. Colloid Interface Sci., 2015, 451, 125-133.

8 R. Li, Y. Wu, L. Shen, J. Chen and H. Lin, J. Colloid Interface Sci., 2018, 531, 493-501.

9 P. F. Andrade, A. F. de Faria, S. R. Oliveira, M. A. Z. Arruda and M. do C. Gonçalves, Water Res., 2015, 81, 333-342.

10 B. B. Campos, L. Gelde, M. Algarra, J. C. G. Esteves da Silva, M. I. Vázquez and J. Benavente, Carbohydr. Polym., 2016, 151, 939-946.

11 J. Kim, Q. Fu, K. Xie, J. M. P. Scofield, S. E. Kentish and G. G. Qiao, J. Membr. Sci., 2016, 515, 54-62.

12 V. Boffa, L. Parmeggiani, A. H. Nielsen and G. Magnacca, Microporous Mesoporous Mater., 2016, 221, 81-90.

13 Z. Sun and F. Chen, Int. J. Biol. Macromol., 2016, 91, 143-150. 14 J. Li, X. Liu, J. Lu, Y. Wang, G. Li and F. Zhao, J. Colloid Interface Sci., 2016, 484, 107-115.

15 Z. Chen, X. Qin, Q. Zhang, Y. Li and H. Wang, J. Colloid Interface Sci., 2015, 459, 44-52.

16 F. Diagne, R. Malaisamy, V. Boddie, R. D. Holbrook, B. Eribo and K. L. Jones, Environ. Sci. Technol., 2012, 46, 4025-4033.

17 M. S. Mauter, Y. Wang, K. C. Okemgbo, C. O. Osuji, E. P. Giannelis and M. Elimelech, ACS Appl. Mater. Interfaces, 2011, 3, 2861-2868.

18 S. Agnihotri, S. Mukherji and S. Mukherji, RSC Adv., 2014, 4, 3974-3983.

19 I. Sondi and B. Salopek-Sondi, J. Colloid Interface Sci., 2004, 275, 177-182.

20 X. Hong, J. Wen, X. Xiong and Y. Hu, Environ. Sci. Pollut. Res., 2016, 23, 4489-4497.

21 A. Alshareef, K. Laird and R. B. M. Cross, Appl. Surf. Sci., 2017, 424, 310-315.

22 B. Xue, D. Wang, J. Zuo, X. Kong, Y. Zhang, X. Liu, L. Tu, Y. Chang, C. Li, F. Wu, Q. Zeng, H. Zhao, H. Zhao and H. Zhang, Nanoscale, 2015, 7, 8048-8057.

23 S. Chen and D. L. Carroll, Nano Lett., 2002, 2, 1003-1007.
24 S. Singh, A. Bharti and V. K. Meena, J. Mater. Sci.: Mater. Electron., 2014, 25, 3747-3752.

25 S. Viarbitskaya, A. Teulle, R. Marty, J. Sharma, C. Girard, A. Arbouet and E. Dujardin, Nat. Mater., 2013, 12, 426-432.

26 L. Y. Ng, A. W. Mohammad and C. Y. Ng, Adv. Colloid Interface Sci., 2013, 197-198, 85-107.

27 C. Xue, G. S. Métraux, J. E. Millstone and C. A. Mirkin, J. Am. Chem. Soc., 2008, 130, 8337-8344.

28 E. C. Cho, C. M. Cobley, M. Rycenga and Y. Xia, J. Mater. Chem., 2009, 19, 6317-6320.

29 J. Yin, Y. Yang, Z. Hu and B. Deng, J. Membr. Sci., 2013, 441, 73-82.

30 M. Ben-Sasson, X. Lu, E. Bar-Zeev, K. R. Zodrow, S. Nejati, G. Qi, E. P. Giannelis and M. Elimelech, Water Res., 2014, 62, 260-270.

31 W. Ma, A. Soroush, T. Van Anh Luong, G. Brennan, M. S. Rahaman, B. Asadishad and N. Tufenkji, Water Res., 2016, 99, 188-199.

32 B. Reidy, A. Haase, A. Luch, K. A. Dawson and I. Lynch, Materials, 2013, 6, 2295-2350.

33 S. Pal, Y. K. Tak and J. M. Song, J. Biol. Chem., 2015, 290, 1712-1720.

34 M. Gao, L. Sun, Z. Wang and Y. Zhao, Mater. Sci. Eng., C, 2013, 33, 397-404.

35 J. R. Morones, J. L. Elechiguerra, A. Camacho, K. Holt, J. B. Kouri, J. T. Ramírez and M. J. Yacaman, Nanotechnology, 2005, 16, 2346-2353.

36 T. Nguyen, F. A. Roddick and L. Fan, Membranes, 2012, 2, 804-840.

37 B. D. Cho and A. G. Fane, J. Membr. Sci., 2002, 209, 391-403.

38 C. Y. Tang, T. H. Chong and A. G. Fane, Adv. Colloid Interface Sci., 2011, 164, 126-143.

39 J. H. Li, X. S. Shao, Q. Zhou, M. Z. Li and Q. Q. Zhang, Appl. Surf. Sci., 2013, 265, 663-670.

40 E. M. Thurman, R. L. Wershaw, R. L. Malcolm and D. J. Pinckney, Org. Geochem., 1982, 4, 27-35. 